\title{
Mechanical analysis of vibratory conveyor mechanism
}

\author{
Yerlan Yeleukulov ${ }^{1}$, Alfiya Atalykova ${ }^{1}$, Algazy Zhauyt $^{1, *}$, Zhubanyshbay Abdimuratov $^{1}$, \\ Saltanat Yussupova ${ }^{1}$, Assel Alik ${ }^{3}$, Gaukhar Kenzhebayeva ${ }^{3}$ \\ ${ }^{1}$ Almaty University of Power Engineering and Telecommunications, 050013 Almaty, Kazakhstan \\ ${ }^{2}$ Kazakh National Agrarian University, 050010 Almaty, Kazakhstan \\ ${ }^{3}$ Kazakh Academy of Transport and Communications named after M. Tynyshpayev, 050012 Almaty, \\ Kazakhstan
}

\begin{abstract}
The paper describes some of the basics of kinematics and dynamics of vibratory conveyor drive. Featured are dynamic modes of material that is being transported. Presented are the optimum material handling, and most rational mode. Introducing the empirical dependence transport speed as a function of angle bending conveyor, amplitude of oscillation of the transport container, the oscillation frequency excitation angle at which operates the actuating force and the coefficient mode of operation of the conveyor. Featured are some of the drive vibratory conveyors, and especially the operation of electromagnetic vibratory conveyor. In this paper is studied a vibratory conveyor that is placed on an elastic base. Using the closed contours method it was determined the system that needs to be solved to obtain graphical representation for the generalized coordinates determining the position of the mechanical system elements. The shaking conveyor represents the chase hanged or supported to the fixed section. The chase commits oscillating motions hereupon the cargo which is in the chase, migrates concerning to the chase. The nature of the flow and its parameters are determined by the nature of the oscillating committed by the chase. Justifying the dynamic parameters of the vibratory conveyor and a study of the strain-energy. Installation causes fluctuations fixed tray. Uniformly distributed load on the tray acts in each element of the mechanism. On the basis of the program MSC Adams investigated the strain-energy and kinetic-energy of each link mechanism with results and calculations.
\end{abstract}

\section{Introduction}

The big application in various fields of the industry was received by the vibrating conveyors applied to transportation of hot, poisonous, chemical aggressive cargoes by the supplement of complete tightness of their relocation [1], and also for transportation of the metallic cuttings damped with emulsion and oil, hot earth which has been beaten out from casting forms, small casting, foundry fusion mixture, etc. The vibrating conveyor represents the chase hanged or supported to the fixed section. The chase commits oscillating motions

*Corresponding author: ali84jauit@mail.ru 
hereupon the cargo which is in the chase, migrates concerning to the chase [2]. The nature of the flow and its parameters are determined by the nature of the oscillating committed by the chase. Vibrating conveyors on the conditions of the chase flow and nature of cargo movement are subdivided on inertial (with variable and constant stress of cargo to the chase) in which [3] cargo under the influence of inertia force glides on the chase, and on vibrating in which cargo tears off the chase and migrates along the chase. The vibrating conveyors [4] are widely applied owing to a number of advantages in these latter days. The questions of the kinematic and dynamic study of the vibrating feeder intended for dosing of the fusion mixture loading of the melting furnaces of foundry production are considered in the presented work [5]. The principle of operation of the vibrating conveyor is described, and it is devoted the kinematic analysis of the action. The differential equation of the link move of the reduction of the vibrating conveyor is considered in the difference method (the approximate method) solutions of the equation of move of the vibrating conveyor are resulted [6]. It is devoted to the analysis of the equations solutions of conveyor move [7]. Here tables of the results and relocation drawing and velocity of the leading link depending on time are resulted. Horizontal and vertical path-time processes as well as the movement behavior of the conveyed good were recorded. Due to the joint time basis of the measuring signals, it is possible to interpret the reaction of the conveyed good to certain movement patterns [8]. The vertical movement of the conveyor organ was recorded at two points (on the left and right side) to evaluate the steadiness of the movement pattern over the entire length of the conveyor organ. By this methodology, staggering or tilting movements of the conveyor organ can be detected, and conclusions on suitable measuring points can be drawn [9].

\section{Materials and methods}

It is the action of the III class (fig. 1). The crew BCDE basic crew from which there are three leads $\mathrm{EF}, \mathrm{AB}, \mathrm{DG}$. The link OA is a leading link.

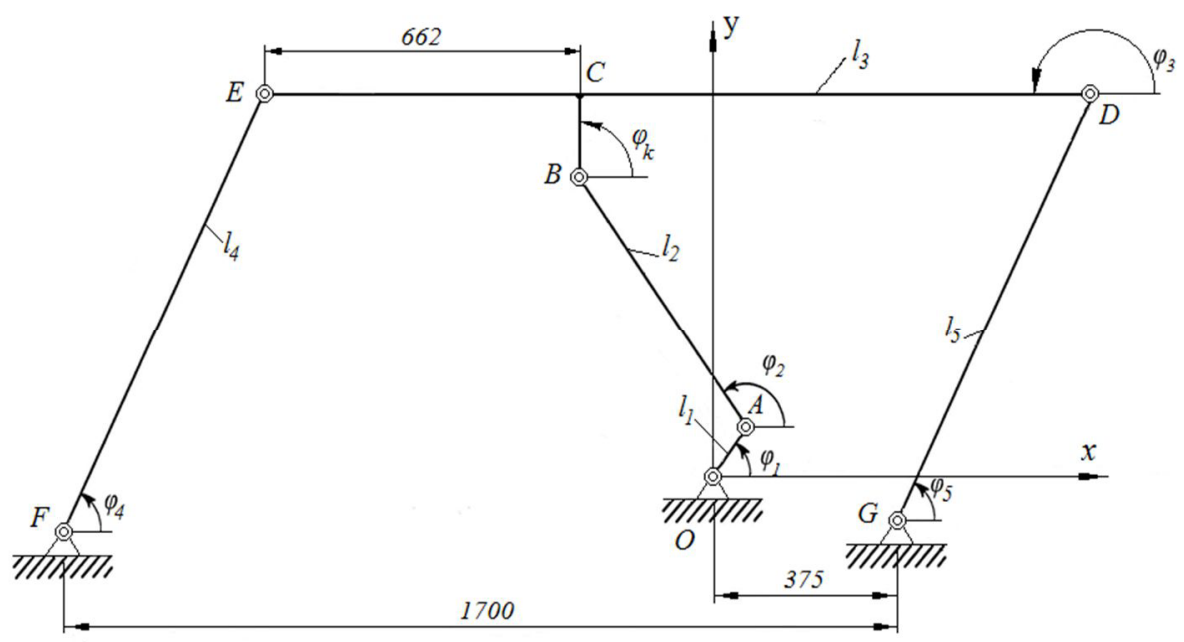

Fig. 1. Kinematic scheme of the vibratory conveyor mechanism. 
Table 1. The action has following performances.

\begin{tabular}{|c|c|c|c|c|c|}
\hline \multirow{2}{*}{ № } & \multicolumn{5}{|c|}{ links } \\
\cline { 2 - 6 } & 1 & 2 & 3 & 4 & 5 \\
\hline Mass, $\mathrm{kg}$ & $m_{1}=30$ & $m_{2}=65$ & $m_{3}=1160$ & $m_{4}=59$ & $m_{5}=59$ \\
\hline Length units, mm & $l_{1}=60$ & $l_{2}=430$ & $l_{3}=1700$ & $l_{4}=440$ & $l_{5}=440$ \\
\hline
\end{tabular}

This action has one axis of motion, therefore relocation, velocities and speed-up of the driven link and action dots are functions of the relocation, velocities and speed-up of the leading link. Therefore we will find analytical dependences between relocations, velocities of the driven links and leading link. As the leading link enters into the rotational pair with the console we set function. By the solution of the action move problem with one degree of freedom we will use the equation of the action move of the engine aggregate $\varphi_{1}=\varphi_{1}(t)$ :

$$
I_{M}\left(\varphi_{1}\right) \frac{d \omega_{1}}{d t}=\frac{\omega_{1}^{2}}{2} \frac{d \mathrm{I}_{M}\left(\varphi_{1}\right)}{d \varphi_{1}}=M_{M}-M_{R}
$$

It is known to us dependence $M_{M}(\omega)$ and $M_{R}\left(\varphi_{1}\right)$. We determine $M_{R}\left(\varphi_{1}\right)$ the resulted moment of resistance forces from power equaling of the resulted moment of force resistance and the sum of powers of the moments [5] of resistance forces of operating in links E, D, F, G:

$$
\begin{gathered}
M_{R} \omega_{1}=M_{E}^{R} \omega_{4}+M_{D}^{R} \omega_{5}+M_{F}^{R} \omega_{4}+M_{G}^{R} \omega_{5} \\
M_{R}=M_{E}^{R} \frac{\omega_{4}}{\omega_{1}}+M_{D}^{R} \frac{\omega_{5}}{\omega_{1}}+M_{F}^{R} \frac{\omega_{4}}{\omega_{1}}+M_{G}^{R} \frac{\omega_{5}}{\omega_{1}}=M_{E}^{R} u_{41}+M_{D}^{R} u_{51}+M_{F}^{R} u_{41}+M_{G}^{R} u_{51}=4 \cdot u_{41} M_{R}
\end{gathered}
$$

Let's find resulted moment of the flywheel actions $I_{M}\left(\varphi_{1}\right)$ from equaling condition of kinematic energy of reduction link to the kinematic energy sum of all links of the action:

$$
\mathrm{I}_{M}\left(\varphi_{1}\right)=\mathrm{I}_{S 1}+m_{2} \vartheta_{S 2 \varphi_{1}}+\mathrm{I}_{S 2} u_{22}^{2}+m_{3} \vartheta_{S 3 \varphi_{1}}^{2}+\left(\mathrm{I}_{4 F}+\mathrm{I}_{5 G}\right) \cdot l_{41}^{2}
$$

We determine inertia moments of the first, second, fourth and fifth links:

$$
\begin{gathered}
\mathrm{I}_{S 1}=m_{1} \cdot \frac{l_{1}^{2}}{2} ; \quad \mathrm{I}_{S 2}=m_{2} \cdot \frac{l_{2}^{2}}{2} ; \quad \mathrm{I}_{4 F}=\mathrm{I}_{S 4}+m_{4} \cdot l_{4}^{\prime}=m_{4} \cdot \frac{l_{4}^{2}}{2}+m_{4} \cdot \frac{l_{4}^{2}}{4} \\
\mathrm{I}_{5 G}=\mathrm{I}_{S 5}+m_{5} \cdot l_{5}^{\prime}=m_{5} \cdot \frac{l_{5}^{2}}{2}+m_{5} \cdot \frac{l_{5}^{2}}{4} \\
M_{R}=M_{R}^{\prime}+K \varphi_{5} ; \quad K=\operatorname{tg} \alpha=\frac{14,86-5,94}{2^{\circ}-1^{\circ}}=\frac{8,92}{0,017453}=511,84 \\
\varphi_{5}=\varphi_{5}\left(\varphi_{1}\right), M_{R}=M_{R}\left(\varphi_{1}\right), M_{R}=-2,98+511,87 \cdot \varphi_{5}, M_{M}=M_{M_{0}}-\alpha_{0} \cdot \omega_{1} \\
\alpha_{0}=\operatorname{tg} \alpha=\frac{2,8-1,8}{1500-150}=\frac{1}{1350}=0,00074 \\
\frac{\mathrm{Y}-\mathrm{Y}_{1}}{\mathrm{Y}_{2}-\mathrm{Y}_{1}}=\frac{\mathrm{X}-\mathrm{X}_{1}}{\mathrm{X}_{2}-\mathrm{X}_{1}} ; \quad \frac{\mathrm{Y}-2,8}{1,8-2,8}=\frac{\mathrm{X}-150}{1500-150}
\end{gathered}
$$




$$
\mathrm{X}=0, \mathrm{Y}=M_{M_{0}}=2,92 \cdot M_{M}=2,92-0,00074 \cdot \omega_{1}
$$

We differentiate the resulted inertia moment on $\varphi_{1}$ : For this purpose we substituted all above found values in the equation:

$$
\begin{aligned}
& \frac{d \mathrm{I}_{M}\left(\varphi_{1}\right)}{d \varphi_{1}}=2 \cdot m_{2} \sqrt{\left(l_{1} \sin \varphi_{1}+l_{2}^{\prime} \sin \varphi_{2} \cdot u_{21}\right)^{2}+\left(l_{1} \cos \varphi_{1}+l_{2}^{\prime} \cos \varphi_{2} \cdot u_{21}\right)^{2}} \cdot \omega_{1} . \\
& \cdot \frac{\left\{\omega_{1}\left[l_{1}\left(\cos \varphi_{1}-\sin \varphi_{1}\right)+l_{2}^{\prime} \cdot u_{21}^{2}\left(\cos \varphi_{2}-\sin \varphi_{2}\right)\right]+l_{2}^{\prime} \frac{d u_{21}}{d \varphi_{1}}\left(\sin \varphi_{2}+\cos \varphi_{2}\right)\right\}}{\sqrt{\left(l_{1} \sin \varphi_{1}+l_{2}^{\prime} \sin \varphi_{2} \cdot u_{21}\right)^{2}+\left(l_{1} \cos \varphi_{1}+l_{2}^{\prime} \cos \varphi_{2} \cdot u_{21}\right)^{2}}}+2 I_{S 2} \frac{l_{1}}{l_{2}} \frac{\sin \left(\varphi_{4}-\varphi_{1}\right)}{\sin \left(\varphi_{2}-\varphi_{1}\right)} . \\
& \cdot \frac{l_{1}}{l_{2}}\left[\frac{u_{41} \cos \left(\varphi_{4}-\varphi_{1}\right)-\cos \left(\varphi_{4}-\varphi_{1}\right)}{\sin \left(\varphi_{4}-\varphi_{2}\right)}-\frac{\left[u_{21} \cos \left(\varphi_{4}-\varphi_{2}\right)-u_{41} \cos \left(\varphi_{4}-\varphi_{2}\right)\right] \cdot \sin \left(\varphi_{4}-\varphi_{1}\right)}{\sin ^{2}\left(\varphi_{4}-\varphi_{2}\right)}\right]+ \\
& +2 m_{3} \omega_{1}^{2}\left[-l_{1}\left(\cos \varphi_{1}+\sin \varphi_{1}\right)-l_{2} \cdot u_{21}^{2}\left(\cos \varphi_{2}-\sin \varphi_{2}\right)+l_{2} \frac{d u_{21}}{d \varphi_{1}}\left(\cos \varphi_{2}-\sin \varphi_{2}\right)+\frac{d u_{51}}{d \varphi_{1}}\left(a \cdot \cos \omega_{5} t-b \cdot \sin \omega_{5} t\right)\right]+ \\
& +2\left(I_{4 F}+I_{5 G}\right) \cdot \frac{l_{1}}{l_{4}}\left\{\frac{\cos \left(\varphi_{1}-\varphi_{2}\right) u_{21}-\cos \left(\varphi_{1}-\varphi_{2}\right)}{\sin \left(\varphi_{2}-\varphi_{4}\right)} \frac{\left[\cos \left(\varphi_{1}-\varphi_{2}\right) u_{21}-\operatorname{co\$ }\left(\varphi_{1}-\varphi_{2}\right) \sin \left(\varphi_{2}-\varphi_{1}\right)\right]}{\sin ^{2}\left(\varphi_{2}-\varphi_{4}\right)}\right\} \frac{l_{1}}{l_{4}} \frac{\sin \left(\varphi_{2}-\varphi_{1}\right)}{\sin \left(\varphi_{2}-\varphi_{4}\right)}= \\
& =2 \cdot m_{2} \cdot \omega_{1}^{2}\left[l_{1} \cdot\left(\cos \varphi_{1}-\sin \varphi_{1}\right)+l_{2}^{\prime} \cdot u_{21}\left(\cos \varphi_{2}-\sin \varphi_{2}\right)+l_{2}^{\prime} \frac{d u_{21}}{d \varphi_{1}}\left(\sin \varphi_{2}-\cos \varphi_{2}\right)\right]+ \\
& +2 \cdot \mathrm{I}_{S 2} \cdot \frac{l_{1}^{2}}{l_{2}^{2}} \frac{\sin \left(\varphi_{4}-\varphi_{1}\right)}{\sin \left(\varphi_{2}-\varphi_{4}\right)}\left\{\frac{u_{41} \cos \left(\varphi_{1}-\varphi_{4}\right)-\cos \left(\varphi_{4}-\varphi_{1}\right)}{\sin \left(\varphi_{2}-\varphi_{4}\right)}-\frac{\left[u_{21} \cos \left(\varphi_{4}-\varphi_{2}\right)-u_{41} \cos \left(\varphi_{4}-\varphi_{2}\right)\right] \sin \left(\varphi_{4}-\varphi_{1}\right)}{\sin ^{2}\left(\varphi_{4}-\varphi_{2}\right)}\right\}- \\
& -2 m_{3} \omega_{1}^{2}\left[l_{1}\left(\cos \varphi_{1}+\sin \varphi_{1}\right)+l_{2}^{\prime} \cdot u_{21}^{2}\left(\cos \varphi_{2}-\sin \varphi_{2}\right)-l_{2} \frac{d u_{21}}{d \varphi_{1}}\left(\sin \varphi_{2}-\cos \varphi_{2}\right)-\frac{d u_{51}}{d \varphi_{1}}\left(a \cdot \cos \omega_{5} t-b \cdot \sin \omega_{5} t\right)\right]+ \\
& +2\left(I_{4 F}+I_{5 G}\right) \cdot \frac{l_{1}^{2}}{l_{4}^{2}} \frac{\sin \left(\varphi_{2}-\varphi_{1}\right)}{\sin \left(\varphi_{2}-\varphi_{4}\right)} \cdot\left\{\frac{\cos \left(\varphi_{1}-\varphi_{2}\right) u_{21}-\cos \left(\varphi_{1}-\varphi_{2}\right)}{\sin \left(\varphi_{2}-\varphi_{4}\right)}-\frac{\left[\cos \left(\varphi_{1}-\varphi_{2}\right) u_{21}-\cos \left(\varphi_{1}-\varphi_{2}\right)\right] \cdot \sin \left(\varphi_{2}-\varphi_{1}\right)}{\sin ^{2}\left(\varphi_{2}-\varphi_{4}\right)}\right\}
\end{aligned}
$$

The move equation of the reduction link of the action looks like:

$$
\begin{aligned}
& \mathrm{I}_{S 1}+m_{2} \cdot \omega_{1}^{2}\left\{\left[\left(l_{1} \sin \varphi_{1}+l_{2}^{\prime} \sin \varphi_{2} \cdot u_{21}\right)^{2}+\left(l_{1} \cos \varphi_{1}+l_{2}^{\prime} \cos \varphi_{2} \cdot u_{21}\right)^{2}\right]+\mathrm{I}_{S 2} \frac{l_{1}^{2}}{l_{2}^{2}} \frac{\sin ^{2}\left(\varphi_{4}-\varphi_{1}\right)}{\sin ^{2}\left(\varphi_{2}-\varphi_{4}\right)}+\right. \\
& \left.+m_{3} \cdot \omega_{1}^{2} \mid\left(-l_{1} \sin \varphi_{1}-l_{2} \sin \varphi_{2} \cdot u_{21}+a \cdot u_{51} \cdot \cos \omega_{5} t\right)^{2}\right]+\left[\left(l_{1} \cos \varphi_{1}+l_{2} \cos \varphi_{2} \cdot u_{21}-b \cdot u_{51} \cdot \sin \omega_{5} t\right)^{2}\right]+ \\
& \left.+\left(\mathrm{I}_{4 F}+\mathrm{I}_{5 G}\right) \frac{l_{1}^{2}}{l_{4}^{2}} \frac{\sin ^{2}\left(\varphi_{2}-\varphi_{1}\right)}{\sin ^{2}\left(\varphi_{2}-\varphi_{4}\right)}\right\} \cdot \frac{d \omega_{1}}{d t}+\frac{\omega_{1}^{2}}{2}\left\{2 \cdot m _ { 2 } \cdot \omega _ { 1 } ^ { 2 } \left[\left(l_{1} \sin \varphi_{1}+l_{2}^{\prime} \sin \varphi_{2} \cdot u_{21}\right) \cdot\left(l_{1} \cos \varphi_{1} \cdot u_{21}^{2}+l_{2}^{\prime} \sin \varphi_{2} \frac{d u_{21}}{d \varphi_{1}}\right)+\right.\right.
\end{aligned}
$$




$$
\begin{gathered}
\left.+\left(l_{1} \cos \varphi_{1}+l_{2}^{\prime} \cos \varphi_{2} \cdot u_{21}\right)\left(-l_{1} \sin \varphi_{1}-l_{2}^{\prime} \sin \varphi_{2} \cdot u_{21}^{2}+l_{2}^{\prime} \cos \varphi_{2} \frac{d u_{21}}{d \varphi_{1}}\right)\right]+ \\
+2 \cdot \mathrm{I}_{S 2} \frac{l_{1}^{2}}{l_{2}^{2}}\left[\frac{\left(\cos \left(\varphi_{1}+\varphi_{4}\right)-\cos \left(\varphi_{1}+\varphi_{4}\right)\right) \sin \left(\varphi_{1}-\varphi_{4}\right)}{\sin ^{2}\left(\varphi_{2}-\varphi_{4}\right)}\right]+2 m_{3} \omega_{1}^{2}\left[\left(-l_{1} \sin \varphi_{1}-l_{2} \sin \varphi_{2} \cdot u_{21}+\right.\right. \\
\left.+a \cdot u_{51} \cos \omega_{5} t\right)\left(-l_{1} \cos \varphi_{1}-l_{2} \cos \varphi_{2} u_{21}^{2}-l_{1} \sin \varphi_{2} \frac{d u_{21}}{d \varphi_{1}}+a \cos \omega_{5} t \frac{d u_{51}}{d \varphi_{1}}\right)+ \\
\left.+\left(l_{1} \cos \varphi_{1}+l_{2} \cos \varphi_{2} \cdot u_{21}-b \cdot u_{51} \sin \omega_{5} t\right) \cdot\left(-l_{1} \sin \varphi_{1}+l_{2} \sin \varphi_{2} \cdot u_{21}^{2}+l_{2} \cos \varphi_{2} \frac{d u_{21}}{d \varphi_{1}}-b \sin \omega_{5} t \frac{d u_{51}}{d \varphi_{1}}\right)\right]+ \\
\left.+\left(\mathrm{I}_{4 F}+\mathrm{I}_{5 G}\right)\left[2 \frac{l_{1}^{2}}{l_{2}^{2}} \cdot \frac{\left[\cos \left(\varphi_{1}+\varphi_{2}\right)-\cos \left(\varphi_{1}-\varphi_{2}\right)\right] \sin \left(\varphi_{2}-\varphi_{1}\right)}{\sin ^{2}\left(\varphi_{2}-\varphi_{4}\right)}\right]\right\}= \\
=M_{M_{0}}-\alpha \cdot \omega_{1}-4 u_{41}\left(M_{R}^{\prime}+K_{0} \varphi_{5}\right)
\end{gathered}
$$

For solution of the Eq. (3) there are following initial conditions: at $t=0, \omega_{1}=0, \varphi_{1}=60^{\circ}$.

\subsection{The approximate method of the equation solutions of the vibrating conveyor}

For constructing on the piece $t \in[0, T]$ of the approximate solution of the move Eq. (3), we copy it in the following kind:

$$
\begin{gathered}
R(t) \frac{d \omega_{1}}{d t}+Q(t) \cdot \omega^{2}(t)=W(t) \\
R(t)=\mathrm{I}_{S_{1}}+\left(m_{2} A(t)+m_{3} H(t) \omega_{1}^{2}+B(t)+D(t)\right. \\
A(t)=l_{1}^{2}+2 l_{1} l_{2}^{\prime}\left(\sin \varphi_{1} \cdot \sin \varphi_{2}+\cos \varphi_{1} \cdot \cos \varphi_{2}\right) \cdot u_{21}+l_{2}^{\prime 2} \cdot u_{21}^{2} \\
\left.+\cos \varphi_{1} \cos \varphi_{2}\right)-2 l_{1} \cdot u_{51}\left(a \cdot \sin \varphi_{1} \cos \omega_{5} t+b \cdot \cos \varphi_{2} \sin \omega_{5} t\right)-2 l_{2} \cdot u_{21}\left(a \cdot \cos \omega_{5} t \sin \varphi_{2}+\right. \\
\left.+b \cdot \sin \omega_{5} t \cdot \cos \varphi_{2}\right) \\
u_{41}^{2}=l_{21}^{2} \cdot u_{21}^{2}+\left(a^{2} \cos ^{2} \omega_{5} t+b^{2} \sin ^{2} \omega_{5} t\right) \cdot u_{51}^{2}+2 l_{1} l_{2} \cdot u_{21}\left(\sin \varphi_{1} \sin \varphi_{2}\right) \\
l_{4} \sin \left(\varphi_{2}-\varphi_{4}\right) \\
B(t)=u_{21}=\frac{l_{1} \sin \left(\varphi_{4}-\varphi_{1}\right)}{l_{2} \sin \left(\varphi_{2}-\varphi_{4}\right)} \\
l_{2}^{2} \sin ^{2}\left(\varphi_{4}-\varphi_{1}\right) \\
D(t)=\left(\mathrm{I}_{4 F}+\mathrm{I}_{5 G}\right) \\
l_{1}^{2} \sin ^{2}\left(\varphi_{2}-\varphi_{1}\right) \\
\sin ^{2}\left(\varphi_{2}-\varphi_{4}\right)
\end{gathered}
$$




$$
\begin{gathered}
E(t)=\left(l_{1} \sin \varphi_{1}+l_{2}^{1} \sin \varphi_{2} \cdot u_{21}\right) \cdot\left(l_{1} \cos \varphi_{1}+l_{2}^{1} \cos \varphi_{2} \cdot u_{21}^{2}+l_{2}^{1} \sin \varphi_{2} \frac{d u_{21}}{d \varphi_{1}}\right)+ \\
+\left(l_{1} \cos \varphi_{1}+l_{2}^{1} \cos \varphi_{2} \cdot u_{21}\right) \cdot\left(-l_{1} \sin \varphi_{1}-l_{2}^{1} \sin \varphi_{2} \cdot u_{21}^{2}+l_{2}^{1} \cos \varphi_{2} \frac{d u_{21}}{d \varphi_{1}}\right) \\
F(t)=\left(-l_{1} \sin \varphi_{1}-l_{2} \sin \varphi_{2} \cdot u_{21}+a \cdot u_{51} \cos \omega_{5} t\right) \cdot\left(-l_{1} \cos \varphi_{1}-l_{2} \cos \varphi_{2} \cdot u_{21}^{2}-l_{1} \sin \varphi_{1} \frac{d u_{21}}{d \varphi_{1}}+a \cdot \cos \omega_{5} t \frac{d u_{51}}{d \varphi_{1}}\right)+ \\
+\left(l_{1} \cos \varphi_{1}+l_{2} \cos \varphi_{2} \cdot u_{21}-b \cdot u_{51} \sin \omega_{5} t\right) \cdot\left(-l_{1} \sin \varphi_{1}-l_{2} \sin \varphi_{2} \cdot u_{21}^{2}+l_{2} \cos \varphi_{2} \frac{d u_{21}}{d \varphi_{1}}-b \cdot \sin \omega_{5} t \frac{d u_{51}}{d \varphi_{1}}\right) \\
N(t)=\frac{\left(\cos \left(\varphi_{1}+\varphi_{4}\right)-\cos \left(\varphi_{1}-\varphi_{1}\right)\right) \sin \left(\varphi_{1}-\varphi_{4}\right)}{\sin ^{2}\left(\varphi_{2}-\varphi_{4}\right)} \\
M(t)=\left(\mathrm{I}_{4 F}+\mathrm{I}_{5 G}\right)\left[2 \frac{l_{1}^{2}}{l_{2}^{2}} \frac{\left(\cos \left(\varphi_{1}+\varphi_{2}\right)-\cos \left(\varphi_{1}-\varphi_{2}\right)\right) \sin \left(\varphi_{2}-\varphi_{1}\right)}{\sin ^{2}\left(\varphi_{2}-\varphi_{4}\right)}\right] \\
W(t)=M_{M_{0}}-\alpha \cdot \omega_{1}-4 \cdot u_{41}\left(M_{R}^{\prime}+K_{o} \varphi_{5}\right)
\end{gathered}
$$

\section{Results and discussion}

The system of differential equations modeling the movement of the material particle on the vibrating plate belonging to the conveyor was solved using Zinovyeva method. Our goal was to find the optimal values of some parameters that allow the obtaining of maximum speed of a vibratory movement on the plate. The basis of the program MSC Adams investigated the strain-energy and kinetic-energy of each link mechanism with results and calculations. Vibratory conveyors represent an essential proportion of the feeding technology used for small and medium-sized bulk goods and piece goods. The obtained speed of the conveyed good is considerably dependent on the operating point of a vibratory conveyor and is often determined by the operating frequency, the amplitude and the throwing angle to the horizontal. The use of vibration isolators to reduce the dynamism transferred to the environment may cause movement patterns of the conveyor that make the calculation of the resulting conveying speed according to the known guidelines impossible. Analysis methods of two-dimensional movement patterns are introduced with the help of theoretical and experimental examinations. Further approaches to simulate movement patterns of conveyed goods are introduced.

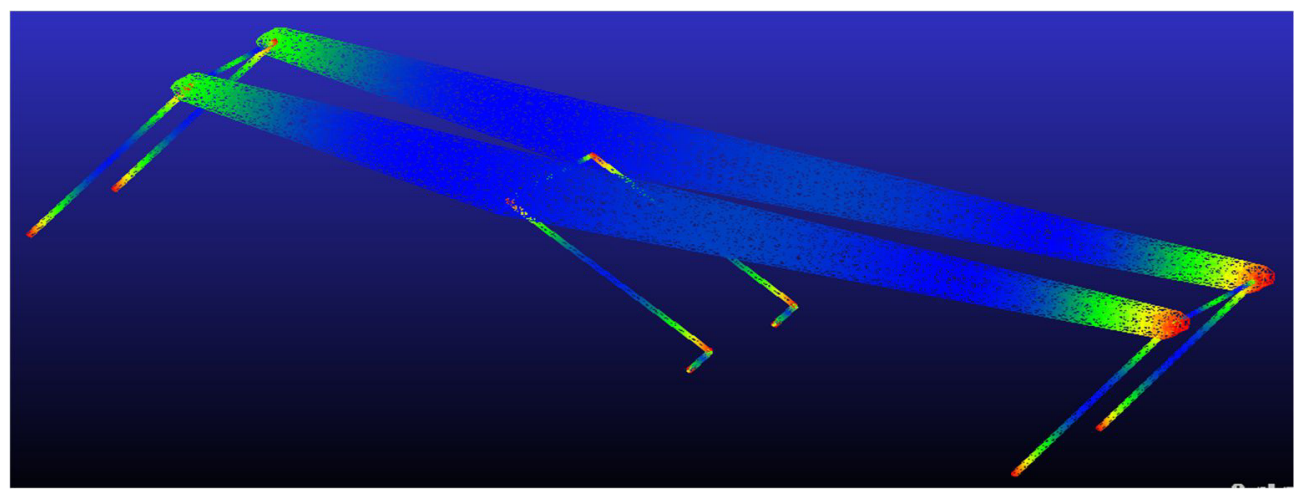

Fig. 2. MSC Adams simulation 3D model of the vibratory conveyor mechanism. 


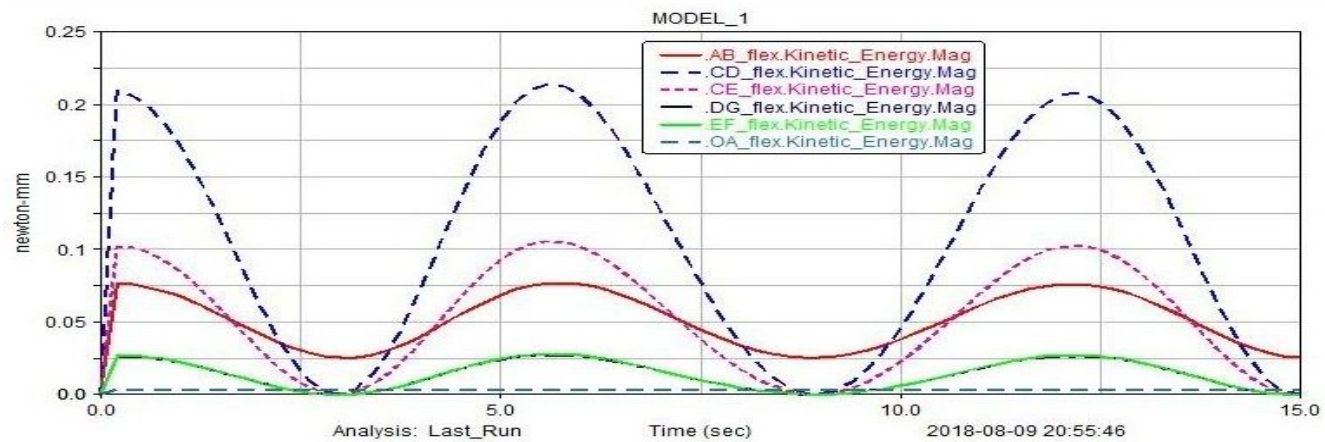

Fig. 3. Computed plot of the kinetic-energy.

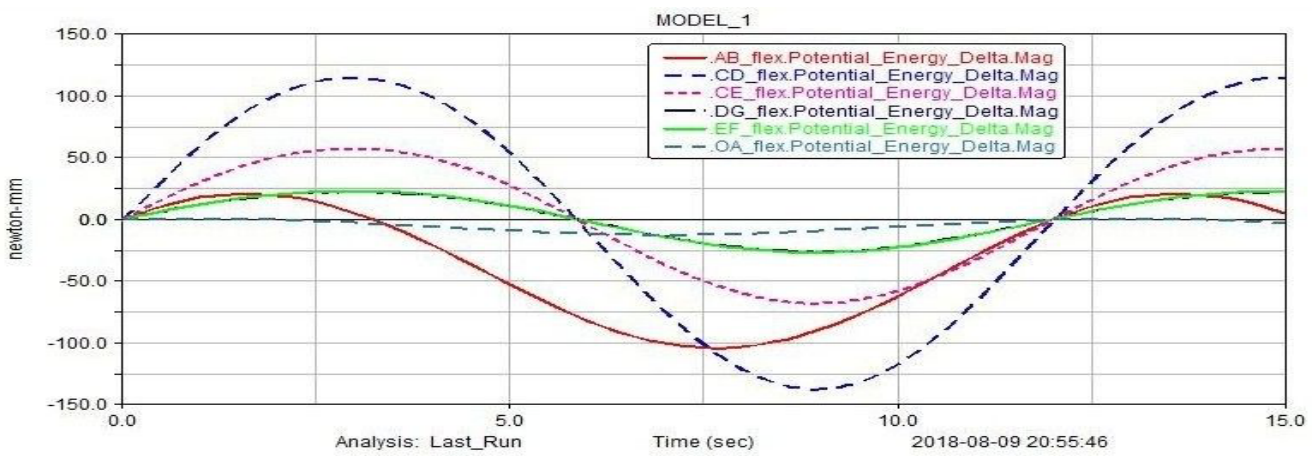

Fig. 4. Computed plot of the potential-energy.

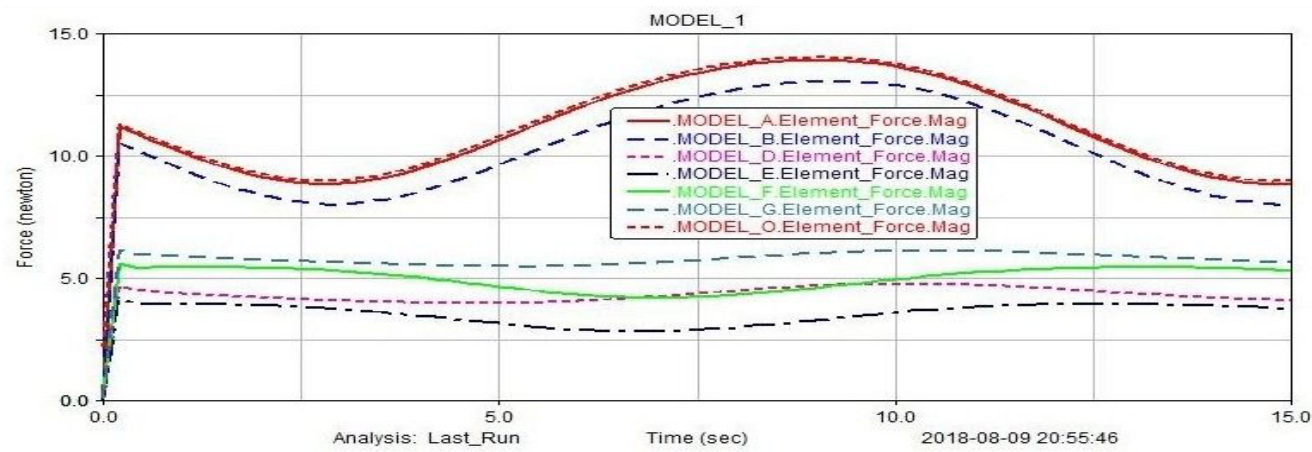

Fig. 5. Computed plot of the element-force.

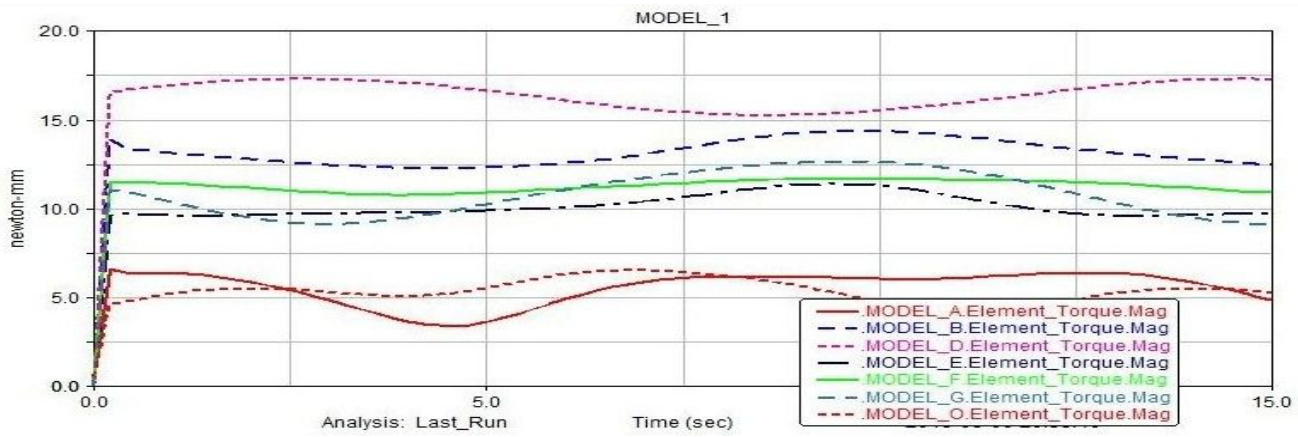

Fig. 6. Computed plot of the element-torque. 


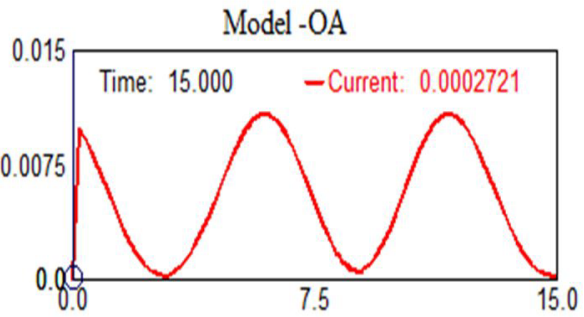

a)

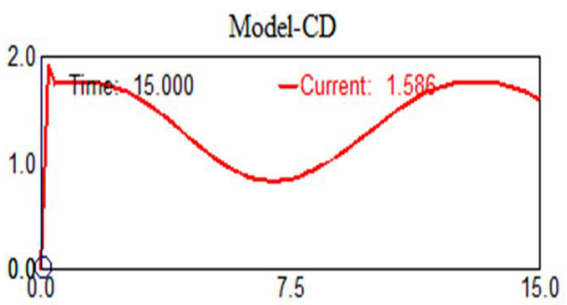

c)

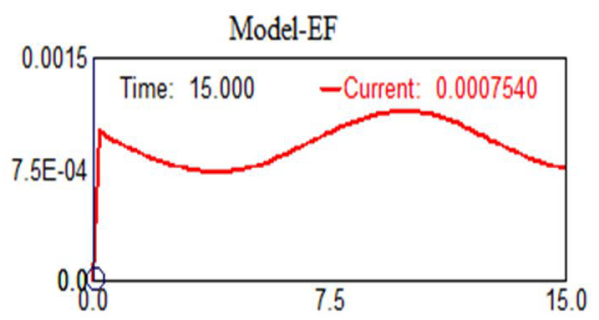

e)

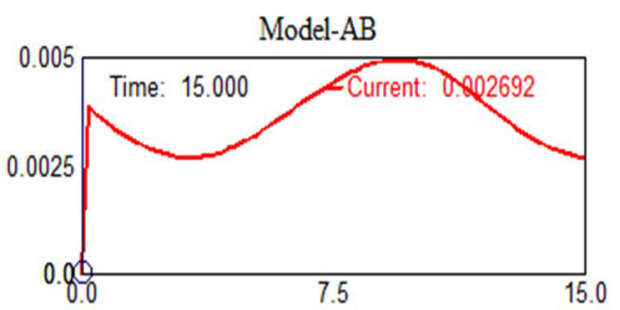

b)

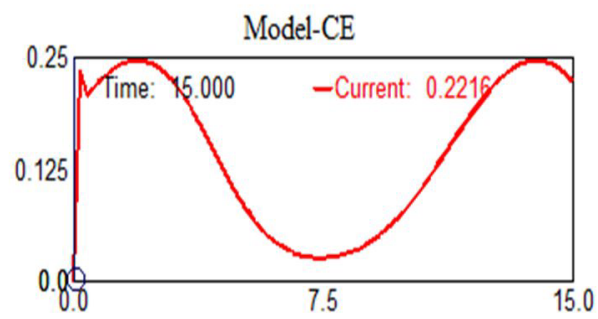

d)

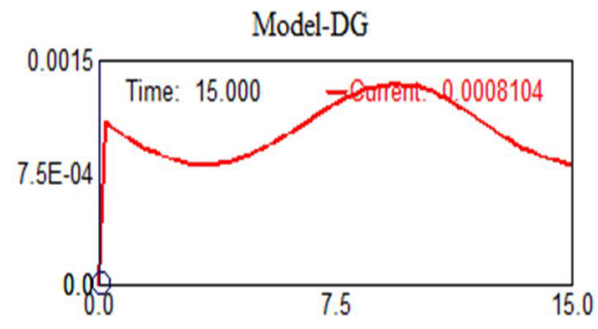

f)

Fig. 7. Computed plot of the strain-energy.

\section{Conclusion}

This experimental data is used for the empirical interpretation as well as for the verification and enhancement of a specially developed mathematic model. On the basis of this mathematic model, a simulation tool should be developed, which will help to calculate conveying speeds more precisely and more detailed than established calculation rules according to the VDI guideline are able to. At the same time, optimal movement rules for oscillating conveyors can be derived. They consequently allow the deliberate development and constructive implementation of efficient vibratory conveyors.

\section{References}

1. V. Andrea, U. Nicolae, A. M. Loana, ACTA Technica napocensis, 4, 55, 949 (2012)

2. A. J. Wyk, J. A. Snyman, P. S. Heyns, N\&O Journal, 1, 10, 12 (1994)

3. C. Yiqing, L. Cuiying, Bulk solid handling, 1, 7, 103 (1987)

4. I. S. Dinu, U. Miorita, U. Nicu, B. Mihai, American Journal of Applied Sciences, 6, 1, 48 (2009) 
5. J. A. Cabrera, A. Simon, M. Prado, Mechanism and Machine theory, 37, 1165 (2002)

6. K. Nendel, T. Risch, Logistics journal, 1, 1, 1 (2010)

7. V. Andrea, U. Nicolae, A. M. Loana, ACTA Technica napocensis, 2, 55, 519 (2012)

8. L. C. Dülger, H. Erdoğan, M. E. Kütük, Int J Intell Syst Appl Eng, 2, 1, 10 (2012)

9. R. R. Bulatovic, S. R. Djordjevic, Theoretical Appl. Mech, 1, 31, 264 (2004) 